\title{
Erratum to: Multivariate normal distribution approaches for dependently truncated data
}

\section{Takeshi Emura • Yoshihiko Konno}

Published online: 12 September 2014

(C) Springer-Verlag Berlin Heidelberg 2014

\section{Erratum to: Stat Papers (2012) 53:133-149 \\ DOI 10.1007/s00362-010-0321-x}

As per the request of Dr. Takeshi Emura, his address has been updated in the affiliation section.

We provide corrections for Emura and Konno (2012). We also numerically verify the corrected formulae. Appendix gives a real data used for numerical analysis.

\section{Correction in the score function [p. 138, definition of $\mathbf{U}_{i}^{*}(\theta)$ ]}

For $\boldsymbol{\theta}^{\prime}=\left(\mu_{L}, \mu_{X}, \sigma_{L}^{2}, \sigma_{X}^{2}\right)$, the corrected score function is

$$
\frac{\partial l(\boldsymbol{\theta})}{\partial \boldsymbol{\theta}}=-n \frac{\dot{c}^{*}(\boldsymbol{\theta})}{c^{*}(\boldsymbol{\theta})}+\sum_{i} \mathbf{U}_{i}^{*}(\boldsymbol{\theta}),
$$

The online version of the original article can be found under doi:10.1007/s00362-010-0321-x.

\section{T. Emura $(\bowtie)$}

Graduate Institute of Statistics, National Central University, Taoyuan, Taiwan e-mail: emura@stat.ncu.edu.tw

\section{Y. Konno}

Japan Women's University, Tokyo, Japan

e-mail: konno@fc.jwu.ac.jp 
Table 1 Calculations of the score function using the three methods

\begin{tabular}{lccc}
\hline$\left(\mu_{L}, \mu_{X}, \sigma_{L}^{2}, \sigma_{X}^{2}\right)$ & Numerical derivative & Corrected formula & Incorrect formula \\
\hline$(10,20,30,40)$ & 2.86657155 & 2.86657152 & -137.0459 \\
$(20,40,60,80)$ & 0.04255583 & 0.04255583 & -279.9137 \\
$(30,60,90,120)$ & -0.10249465 & -0.10249456 & -420.0733 \\
$(40,80,120,160)$ & -0.02560967 & -0.02560980 & -560.0037 \\
\hline
\end{tabular}

The score function is computed by using a read data in Appendix

Numerical derivative: $\left\{l\left(\mu_{L}, \mu_{X}, \sigma_{L}^{2}+h, \sigma_{X}^{2}, 0\right)-l\left(\mu_{L}, \mu_{X}, \sigma_{L}^{2}, \sigma_{X}^{2}, 0\right)\right\} / h$, where $h=10^{-7}$

Corrected formula $=\frac{\partial l(\boldsymbol{\theta})}{\partial\left(\sigma_{L}^{2}\right)}=-n \frac{1}{c^{*}(\boldsymbol{\theta})} \frac{\partial c^{*}(\boldsymbol{\theta})}{\partial\left(\sigma_{L}^{2}\right)}+\sum_{i}\left\{-\frac{1}{2 \sigma_{L}^{2}}+\frac{\left(L_{i}-\mu_{L}\right)^{2}}{2 \sigma_{L}^{4}}\right\}$

Incorrect formula of Emura and Konno (2012)

where

$$
c^{*}(\boldsymbol{\theta})=\Phi\left(\frac{\mu_{X}-\mu_{L}}{\sqrt{\sigma_{X}^{2}+\sigma_{L}^{2}}}\right), \quad \mathbf{U}_{i}^{*}(\boldsymbol{\theta})=\left[\begin{array}{c}
\left(L_{i}-\mu_{L}\right) / \sigma_{L}^{2} \\
\left(X_{i}-\mu_{X}\right) / \sigma_{X}^{2} \\
-/\left(2 \sigma_{L}^{2}\right)+\left(L_{i}-\mu_{L}\right)^{2} /\left(2 \sigma_{L}^{4}\right) \\
-/\left(2 \sigma_{X}^{2}\right)+\left(X_{i}-\mu_{X}\right)^{2} /\left(2 \sigma_{X}^{4}\right)
\end{array}\right] .
$$

The error occurred in the third and fourth components of $\mathbf{U}_{i}^{*}(\boldsymbol{\theta})$.

To confirm that Eqs. (1) and (2) are correct, we focus on the third component of Eq. (1):

$$
\frac{\partial l(\boldsymbol{\theta})}{\partial\left(\sigma_{L}^{2}\right)}=-n \frac{1}{c^{*}(\boldsymbol{\theta})} \frac{\partial c^{*}(\boldsymbol{\theta})}{\partial\left(\sigma_{L}^{2}\right)}+\sum_{i}\left\{-\frac{1}{2 \sigma_{L}^{2}}+\frac{\left(L_{i}-\mu_{L}\right)^{2}}{2 \sigma_{L}^{4}}\right\} .
$$

We compute the score functions using a real data from Appendix. Then, compare Eq. (3) with the numerical derivative

$$
\left\{l\left(\mu_{L}, \mu_{X}, \sigma_{L}^{2}+h, \sigma_{X}^{2}, 0\right)-l\left(\mu_{L}, \mu_{X}, \sigma_{L}^{2}, \sigma_{X}^{2}, 0\right)\right\} / h,
$$

where $h=10^{-7}$. The results are given in Table 1 . We see that there is virtually no difference between the corrected formula and the numerical derivative. On the other hand, the values of the formula of Emura and Konno (2012) are remarkably different from those of the numerical derivative.

\section{Correction in the function $\dot{w}(c)$ [p. 139]}

Emura and Konno (2012) considered a function $w(\cdot):(0,1) \rightarrow[0,1]$, defined as

$$
w(c)=\frac{\boldsymbol{\Phi}^{-1}(c) \phi\left\{\boldsymbol{\Phi}^{-1}(c)\right\}}{c}+\frac{\phi\left\{\boldsymbol{\Phi}^{-1}(c)\right\}^{2}}{c^{2}} .
$$


Table 2 Numerical calculations of the functions $w(c)$ and $\dot{w}(c)$

\begin{tabular}{lrllllllll}
\hline$c$ & 0.1 & 0.2 & 0.3 & 0.4 & 0.5 & 0.6 & 0.7 & 0.8 & 0.9 \\
\hline$w(c)$ & 0.830 & 0.781 & 0.735 & 0.688 & 0.636 & 0.577 & 0.507 & 0.416 & 0.287 \\
$\dot{w}(c)$ Numerical & -0.550 & -0.464 & -0.460 & -0.489 & -0.546 & -0.638 & -0.785 & -1.046 & -1.631 \\
$\dot{w}(c)$ Eq. (4) & -0.550 & -0.464 & -0.460 & -0.489 & -0.546 & -0.638 & -0.785 & -1.046 & -1.631 \\
\hline
\end{tabular}

We define $w(c)=\frac{\boldsymbol{\Phi}^{-1}(c) \phi\left\{\boldsymbol{\Phi}^{-1}(c)\right\}}{c}+\frac{\phi\left\{\boldsymbol{\Phi}^{-1}(c)\right\}^{2}}{c^{2}}$

Numerical derivative is $\dot{w}(c)=\{w(c+h)-w(c)\} / h$, where $h=10^{-7}$

Equation (4) gives expression of $\dot{w}(c)$

They showed that $w$ is strictly decreasing, reflecting the decreasing loss of information at inclusion probability $c$. However, they do not give the formula of $\dot{w}(c)=d w(c) / d c$, and their claim $\dot{w}(1 / 2)=\sqrt{2 / \pi}(1-4 / \pi)$ is incorrect.

Here we provide an explicit derivative given by

$$
\dot{w}(c)=\frac{\left\{1-\boldsymbol{\Phi}^{-1}(c)^{2}\right\} c-\boldsymbol{\Phi}^{-1}(c) \phi\left\{\boldsymbol{\Phi}^{-1}(c)\right\}}{c^{2}}-\frac{2 \phi\left\{\boldsymbol{\Phi}^{-1}(c)\right\}}{c^{3}}\left[c \boldsymbol{\Phi}^{-1}(c)+\phi\left\{\boldsymbol{\Phi}^{-1}(c)\right\}\right]
$$

With this formula, one has

$$
\dot{w}(1 / 2)=\frac{1}{1 / 2}-\frac{1}{2 \pi(1 / 2)^{4}}=2-\frac{8}{\pi} \cong-0.5464791 .
$$

We have confirmed the correctness of Eqs. (4) and (5) in Table 2.

Acknowledgments This work was financially supported by the National Science Council of Taiwan (NSC101-2118-M008-002-MY2). The authors thank Pan Chi-Hung for finding and correcting the errors in our paper.

\section{Appendix: Data on scores from examination and homework (Chung 2013)}

The example is due to Chung (2013). Table 3 records two evaluation items: $X^{O}=$ score from examination and $Y^{O}=$ score from homework are recorded for 10 students. Define a weighted mean $Z^{O}=0.75 X^{O}+0.25 Y^{O}$. Students receive a "fair" if $Z^{O} \geq 60$ and a "fail" if $Z^{O}<60$, respectively. Suppose that a teacher can obtain samples for those students with $Z^{O} \geq 60$. Then, $Z^{O} \geq 60$ is equal to $X^{O} \geq L^{O}$, where $L^{O}=80-Y^{O} / 3$. Accordingly, the observed data is $\left(L_{j}, X_{j}\right)$, subject to $L_{j} \leq X_{j}$, for $j=1,2, \ldots, 7$ in which 7 out of 10 samples are included. 
Table 3 Test score data for two items $X^{O}=$ score from examination, and $Y^{O}=$ score from homework for 10 students

\begin{tabular}{llrrrrrrrrr}
\hline Student ID & 1 & \multicolumn{1}{c}{2} & 3 & 4 & 5 & 6 & 7 & 8 & 9 & 10 \\
\hline$X^{O}$ & 95 & 100 & 40 & 45 & 60 & 75 & 55 & 60 & 70 & 90 \\
$Y^{O}$ & 65 & 30 & 100 & 50 & 70 & 80 & 40 & 70 & 85 & 85
\end{tabular}

Note The scores for ID = 1, 2 and 3 are taken from Chung (2013). The scores for ID = 4-10 are randomly generated

\section{References}

Chung H (2013) Application of approximate reasoning using triangular and sine-curved membership functions. In: Balas VE et al (eds) New concepts and applications in soft computing. Springer-Verlag, Berlin, pp 141-155

Emura T, Konno Y (2012) Multivariate normal distribution approaches for dependently truncated data. Stat Papers 53:133-149 